\title{
INVERTEBRADOS DEL INTERMAREAL ROCOSO DEL DEPARTAMENTO DE LIMA, PERÚ: UNA LISTA COMENTADA DE ESPECIES
}

\section{THE ROCKY SHORE INVERTEBRATES FROM LIMA DEPARTMENT, PERU: A COMIMENTED LIST OF SPECIES}

\author{
Carlos Paredes ${ }^{1}$, Franz Cardoso ${ }^{1}$ y Juan Tarazona ${ }^{2}$
}

\section{RESUMIEN}

La diversidad faunística de la zona intermareal rocosa del departamento de Lima, Perú, fue estudiada en 16 localidades, con énfasis en la dominancia relativa de las especies y su distribución vertical. Se registró 175 especies de invertebrados incluidos en 11 Phyla, y con predominio de los moluscos (79 especies), crustáceos (44 especies) y poliquetos (30 especies), los tres taxa que caracterizan el bentos marino. Considerando los estudios conocidos, el $41 \%$ del total de las especies está presente en la comunidad de Perumytilus purpuratus, $34 \%$ en Semimytilus algosus y $50 \%$ en Phragmatopoma moerchi. La diversidad y la zonación es generalizada para la Provincia Peruana.

Palabras clave: Invertebrados, diversidad, orilla rocosa, Lima, Perú.

\section{ABSTRACT}

The faunistic diversity of the rocky intertidal zone of the Department of Lima, Peru, was studied in sixteen localities with emphasis in the relative dominance of the species and its vertical distribution. 175 species of invertebrates are registered, predominating the mollusks (79 species), crustaceans (44 species) and polychaete (30 species), the three taxa that predominate in the benthos marine. Considering the communities studied $41 \%$ for Perumytilus purpuratus are registered, $34 \%$ in Semimytilus algosus and $50 \%$ of the species for Phragmatopoma moerchi. Diversity and zonation are generalized for the Peruvian Province.

Key words: Invertebrates, diversity, rocky shore, Lima, Perú.

\section{INTRODUCCIÓN}

La diversidad faunistica de la zona intermareal rocosa del litoral del Departamento de I ima fue estudiada intensivamente entre 1970 y 1974. Trabajos posteriores se han centralizado en La Bahía de Ancón y continuado en otros lugares, dentro de los proyectos "Estudio de las Comunidades Bentónicas del Departamento de Lima”, "El

1 Lab. de Invertebrados Acuáticos, Facultad de Ciencias Biológicas, UNMSM, Apdo. 11-0058, Lima 11, Perú.

2 Lab. de Ecología Marina, Facultad de Ciencias Biológicas, UNMSM, Apdo. 1898, Lima 100, Perú.
Bentos y su interacción en el ecosistema marino somero" y "Respuestas del ecosistema marino somero a la oscilación sureña 'El Niño' y al cambio global". Las investigaciones en la zona intermareal rocosa comprenden: La zonación biocenológica de la orilla rocosa (Paredes, 1974b), la caracterización de las comunidades de mitílidos (Paredes y Tarazona, 1980), la comunidad de Phragmalopoma moerchi (Pardo, 1975), y el inventario de los invertebrados (Paredes, 1974a). El objetivo de este trabajo ha sido integrar los registros dispersos en la literatura científica y colecciones locales, y hacerlos disponibles en una lista, incluyendo comentarios ecológicos. 


\section{MATERIAL Y NIÉTODOS}

La investigación se llevó a cabo en el litoral rocoso del Departamento de Lima ( $10^{\circ} 45^{\prime}$ a $13^{\circ} 01^{\prime} \mathrm{S}$ ), habiéndose trabajado en dieciséis localidades. Los lugares principales (Figura 1) fueron, de Norte a Sur, los siguientes: Barranca (1), Carquín (2), Chancaíllo (3), Ancón (4), La Herradura (5), Punta Negra (6), San Bartolo (7), Pucusana (8), Asia (9), Cerro Azul (10); y otros lugares fueron: $\mathrm{Me}$ dio Mundo (11), Isla San Lorenzo (12), Lima (13), La Punta (14), Agua Dulce (15), y Embajadores (16).

De 1970 a 1974 se realizaron muestreos intensivos en los lugares señalados, y posteriormente se ha continuado trabajando con regularidad en la Bahía de Ancón, y con menor frecuencia en las otras localidades, con la finalidad de realizar un inventario de los inver- tebrados marinos del Departamento de Lima. En lo posible, se tomaron datos sobre las relaciones entre las diversas especies y sobre la densidad de las poblaciones o dominancia relativa, utilizando la siguiente escala: rara, escasa, frecuente, común, dominante. Para lo cual nos basamos en las escalas ideadas por Crisp \& Southward, Ballantine, y Moyse \& Nelson-Smith (citadas por Tait, 1971), que son útiles para registrar el número aproximado de algunos organismos del litoral.

Para el estudio de la zonación bioecológica y caracterización de la misma, nos hemos basado en la nomenclatura zonal propuesta por Paredes (1974b): Franja supralitoral (FS), Zona mediolitoral (ZM), Zona mediolitoral superior (ZMS), Zona mediolitoral media (ZMM), Zona mediolitoral inferior (ZMI), Franja infralitoral (FS), Zona infralitoral (ZI).

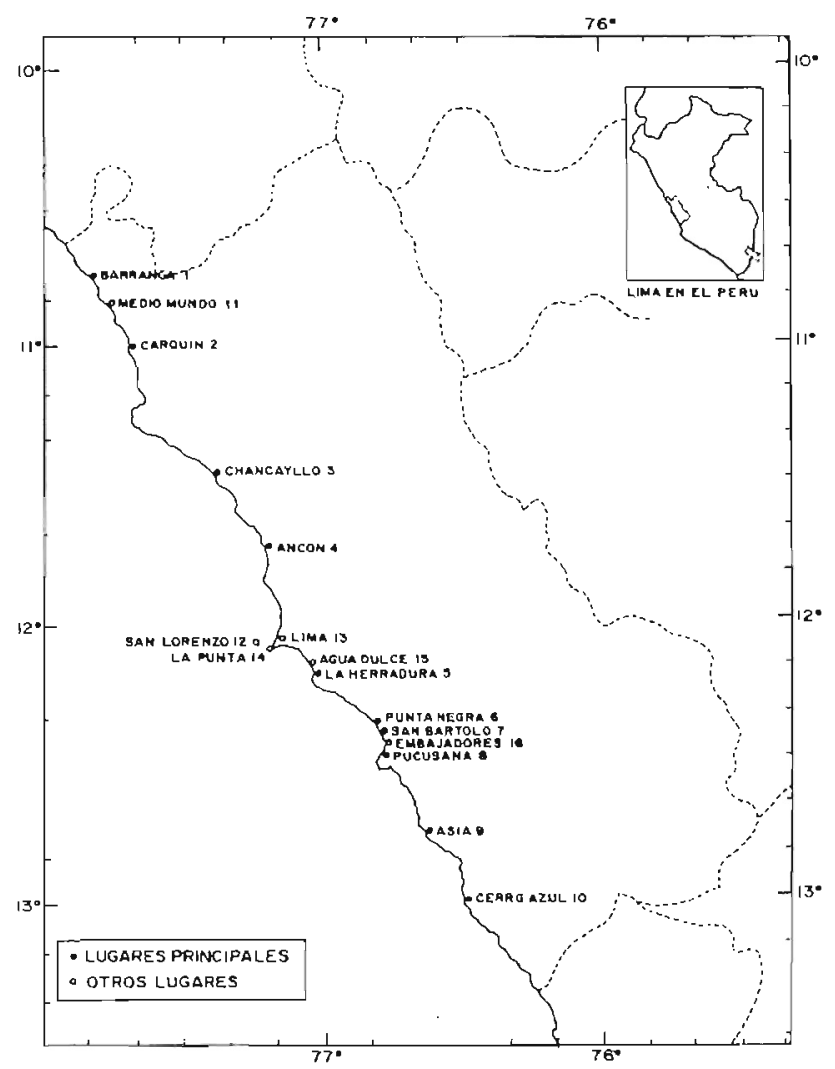

Figura 1. Localidades de muestreo en el Departamento de Lima. 


\section{RESULTADOS}

Una revisión de la literatura (Paredes, 1974a; Huamán, 1974; Tarazona, 1974; Pardo, 1975; Paredes, 1980, 1986; Tarazona et al., 1985) sobre los invertebrados del litoral rocoso del Departamento de Lima y del material existente en el Laboratorio de Invertebrados Acuáticos de la Facultad de Ciencias Biológicas y el Departamento de Malacología y Carcinología del Museo de Historia Natural de la Universidad Nacional Mayor de San Marcos, colectados durante 25 años, nos ha permitido determinar 175 especies y 126 géneros de invertebrados marinos (Tabla 1), pertenecientes a II Phyla y agrupados en 39 órdenes y 76 familias. La mayoría fueron moluscos (79 especies), de los cuales 57 son gairirópodos. Los crustáceos estuvieron bien representados (44 especies), seguidos por los poliquetos ( 30 especies), los tres taxa (Figura 2) que predominan en el bentos marino. De acuerdo a la dominancia relativa resultaron dominantes tres especies (Perumytilus purpuratus, Semimytilus algosus, Jehlius cirratus), 52 fueron comunes, 51 frecuentes, 38 escasas y raras 31 especies.

En la tabla 2 se menciona el número de especies registradas de algunas localidades: Ancón (151), Pucusana (113), Asia (79) y Chancaíllo (60), observándose que el mayor número se presenta en las orillas protegidas y semiexpuestas. En relación con las comunidades de mitílidos, el $34 \%$ de las especies está presente en la comunidad de Semimytilus algosus y el $41 \%$ en la comunidad de Perumytilus purpuratus. El 50\% de las especies está representada en la comunidad de Phragmatopoma moerchi.

La mayoría de los grupos y el $58 \%$ de las especies están distribuidos en la zona mediolitoral. El 28,7\% de estas especies, además de la zona mediolitoral, también habita la franja supralitoral y/o infralitoral. De todos los Phyla, el $54,5 \%$ de las especies de equinodermos y el $45 \%$ de moluscos fueron encontrados sólo en la franja infralitoral y/o zona infralitoral.

\section{DISCUSIÓN y CONCLUSIONES}

La diversidad de invertebrados bentónicos en las áreas costeras del Perú está pobremente estudiada (Tarazona \& Valle, 1998) en comparación con los inventarios de vertebrados. Entre los pocos inventarios realizados en el intermareal rocoso, podemos mencionar los de Paredes (1974a) que reportó 125 especies para el Departamento de Lima y posteriormente Paredes et al., (1988) registran 156 especies en el área de Pisco, distribuidas en

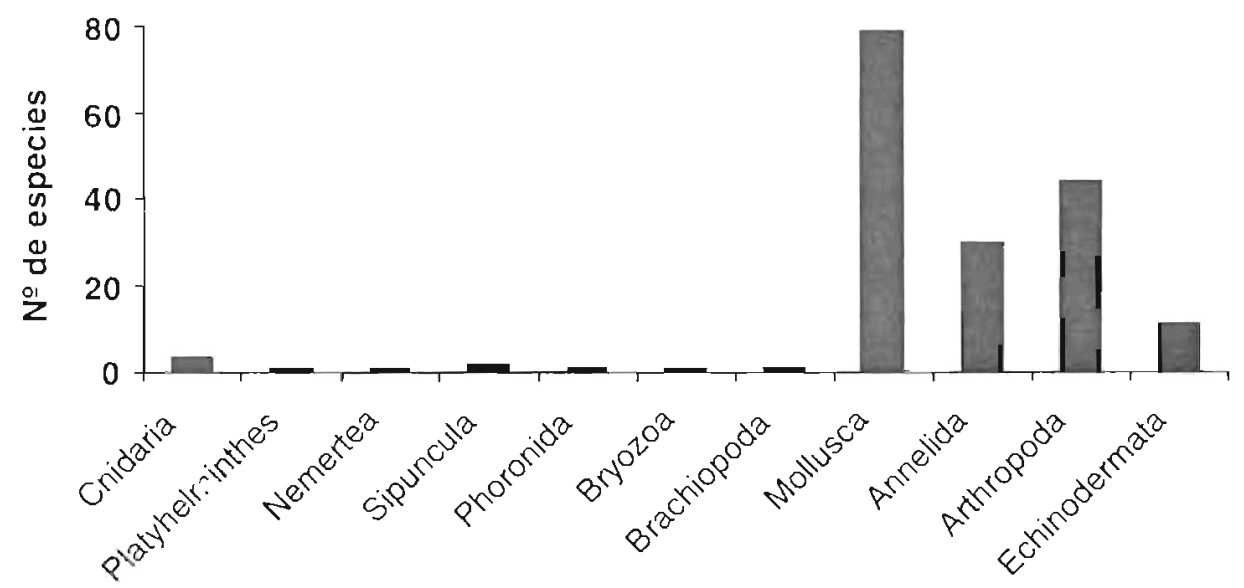

Figura 2. Diversidad por grupos taxonómicos de invertebrados del intermareal rocoso del Departamento de Lima. 
Tabla1. Invertebrados del intermareal rocoso del Departamento de Lima, Perú

(a Los números indican las localidades señaladas en la Figura 1; * Especies que llegaron con El Niño; " Nuevos registros para el Perú)

\begin{tabular}{|c|c|c|c|}
\hline PHYLUM: Clase & Localidadª & Dominancia & Zonación \\
\hline \multicolumn{4}{|l|}{ CNIDARIA: Anthozoa } \\
\hline Anthothoe chilensis (Lesson, 1830) & 4,8 & Frecuente & $\mathrm{Fl}, \mathrm{Zl}$ \\
\hline Phyllactis concinnata Dana, 1846 & $1,3,4,6,9$ & Frecuente & $\mathrm{ZMI}, \mathrm{FI}$ \\
\hline Phymactis clematis (Drayton, 1846) & $1,2,3,4,6,8,9,10$ & Común & $\mathrm{ZMI}, \mathrm{Fl}$ \\
\hline Phymanthea pluvia (Drayton, 1846) & $1,2,3,4,6,8,9,10$ & Escasa & $\mathrm{ZM}, \mathrm{FI}$ \\
\hline \multicolumn{4}{|l|}{ PLATYHELMINTHES: Turbellaria } \\
\hline \multicolumn{3}{|l|}{ NEMERTEA: Enopla } & $\mathrm{ZM}, \mathrm{FI}$ \\
\hline \multicolumn{3}{|l|}{ SIPUNCULA: Sipunculidea } & $\triangle M$ \\
\hline \multicolumn{3}{|l|}{ SIPUNCULA: Phascolosomatidea } & ZMI \\
\hline $\begin{array}{l}\text { Apionsoma (A.) misakianum Ikeda, } 1904 \\
\text { PHORONIDA }\end{array}$ & 4 & Rara & $\triangle M M$ \\
\hline Phoronis ovalis Wright, 1856 & 4 & Rara & $\mathrm{FI}$ \\
\hline \multicolumn{4}{|l|}{ BRYOZOA: Gymnolaemata } \\
\hline Bugula neritina (Linnaeus, 1758) & $7,8,9$ & Frecuente & $\mathbb{Z M}$ \\
\hline \multicolumn{4}{|l|}{ BRACHIOPODA: Inarticulata } \\
\hline $\begin{array}{l}\text { Discinisca lamellosa (Broderip, 1833) } \\
\text { MOLLUSCA: Polyplacophora }\end{array}$ & $2,4,8$ & Frecuente & $\mathrm{Fl}, \mathrm{Zl}$ \\
\hline Acanthopleura echinata (Barnes, 1824) & $1,2,3,4,6,8,9,10$ & Común & $\mathrm{ZMI}, \mathrm{Fl}$ \\
\hline Acanthopleura nigra (Barnes, 1824) & $1,2,3,4,6,8,9,10$ & Común & $\mathrm{ZMI}, \mathrm{FI}$ \\
\hline Callistochiton viviparus Plate, 1902 & $1,2,4,6,8,9$ & Frecuente & ZMM \\
\hline Chaetopleura hennahi (Sowerby, 1832) & 4,8 & Frecuente & $\mathrm{Zl}$ \\
\hline Chaetopleura peruviana (Lamarck, 1819) & 4,8 & Frecuente & $\mathrm{FI}, \mathrm{ZI}$ \\
\hline Chiton cumingsii Frembly, 1828 & $1,2,3,4,6,8,9,10$ & Común & $\mathrm{ZMI}, \mathrm{FI}$ \\
\hline Chiton granosus Frembly, 1828 & $1,2,3,4,6,8,9,10$ & Común & ZMS \\
\hline \multicolumn{4}{|l|}{ MOLLUSCA: Gastropoda } \\
\hline Acanthina brevidentata (Wood, 1828)* & 4 & Rara & $\triangle M$ \\
\hline Aplysia (A.) nigra Orbigny, 1837 & 1,4 & Escasa & $\mathrm{Fl}, \mathrm{Zl}$ \\
\hline Aplysia (V.) inca Orbigny, 1837 & 1,4 & Escasa & $\mathrm{Fl}, \mathrm{Zl}$ \\
\hline Caecum chilense Stuardo, 1962 & $1,4,6,8,9$ & Común & $\mathrm{ZMM}, \mathrm{ZMI}$ \\
\hline Calyptraea (T.) trochiformis (Born, 1778) & $4,8,9$ & Frecuente & $\mathrm{ZI}$ \\
\hline Cancellaria (B.) chrysostoma Sowerby, $1832^{*}$ & 4 & Rara & $\mathrm{Z} \mid$ \\
\hline Columbella fuscata Sowerby, $1832^{*}$ & 4 & Rara & $\mathrm{Fl}$ \\
\hline Concholepas concholepas (Bruguiere, 1789) & $1,4,8,9,10$ & Frecuente & $\mathrm{Zl}$ \\
\hline Costoanachis? berryi Shasky, 1970 & 13 & Rara & $\triangle M$ \\
\hline Costoanachis nigricans (Sowerby, 1844) & 4 & Común & $\mathrm{Fl}, \mathrm{Zl}$ \\
\hline Crassilabrum crassilabrum (Sowerby, 1834) & $4,8,9$ & Frecuente & $\mathrm{FI}$ \\
\hline Crepidula nivea C.B. Adams, 1852 & 4 & Escasa & $\triangle M$ \\
\hline Crepipatella dilatata (Lamarck, 1822) & $2,4,8,9$ & Común & $\mathrm{ZI}$ \\
\hline Crucibulum (C.) quiriquinae (Lesson, 1830) & $8,12,16$ & Escasa & $\mathrm{ZI}$ \\
\hline Fissurella (C.) asperella Sowerby, $1835^{*}$ & 4 & Rara & $\mathrm{ZI}$ \\
\hline
\end{tabular}




\begin{tabular}{|c|c|c|c|}
\hline PHYLUM: Clase & Localidad ${ }^{\mathrm{a}}$ & Dominancia & Zonación \\
\hline Fissurella (F.) bridgesii Reeve, 1849 & $1,4,8$ & Frecuente & ZMI, FI \\
\hline Fissurella (F.) costata Lesson, 1831 & 4,8 & Común & $\mathrm{FI}, \mathrm{ZI}$ \\
\hline Fissurella (F.) crassa Lamarck, 1822 & $2,3,4,8,9$ & Escasa & $\mathrm{ZMI}, \mathrm{FI}, \mathrm{ZI}$ \\
\hline Fissurella (F.) latimarginata Sowerby, 1835 & $5,7,8$ & Escasa & $\mathrm{ZMI}, \mathrm{FI}, \mathrm{ZI}$ \\
\hline Fissurella (F.) limbata Sowerby, 1835 & $4,6,8,9$ & Escasa & $\mathrm{ZMM}, \mathrm{ZMI}, \mathrm{FI}, \mathrm{Zl}$ \\
\hline Fissurella $(F)$ maxima Sowerby, 1835 & $4,5,6,9$ & Escasa & $\mathrm{FI}, \mathrm{ZI}$ \\
\hline Fissurella (F.) peruviana Lamarck, 1822 & 4,8 & Frecuente & $\mathrm{ZMI}, \mathrm{FI}, \mathrm{ZI}$ \\
\hline Fissurella $(F)$ pulchra Sowerby, 1835 & $4,7,8,9,12$ & Frecuente & $\mathrm{FI}, \mathrm{ZI}$ \\
\hline Hexaplex brassica $\left(\right.$ Lamarck, 1822) ${ }^{\star}$ & 12 & Rara & $\mathrm{Zl}$ \\
\hline Iselica carotica Marincovich, 1973 & $1,2,4,6,8,9$ & Frecuente & $\mathbb{Z M}$ \\
\hline Lucapinella crenifera (Sowerby, 1835) & 7,8 & Escasa & $\mathrm{ZMl}$ \\
\hline Linatella wiegmanni (Anton, 1839)* & 8 & Rara & $\mathrm{Z} \mid$ \\
\hline Nodilittorina araucana Orbigny, 1840 & 9 & Frecuente & $\mathrm{FS}, \mathrm{ZM}$ \\
\hline Nodtlittorina peruviana (Lamarck, 1822) & $1,2,3,4,6,8,9,10$ & Común & $\mathrm{FS}, \mathrm{ZM}$ \\
\hline Lottia araucana (Orbigny, 1839) & 8 & Escasa & $Z M$ \\
\hline Lottia ceciliana (Orbigny, 1841) & $2,3,4,5,8,9$ & Común & FS,ZMS,ZMM \\
\hline Lottia orbignyi (Dall, 1909) & $1,2,3,4,6,8,9,10$ & Escasa & FS,ZMS \\
\hline Mitrella buccinoides (Sowerby, 1832) & $2,4,8$ & Frecuente & $\mathrm{Zl}$ \\
\hline Mitrella unifasciata (Sowerby, 1832) & 4 & Escasa & $\mathrm{ZI}$ \\
\hline Nassarius dentifer (Powys, 1835) & $4,8,9$ & Frecuente & $\mathrm{FI}, \mathrm{Zl}$ \\
\hline Nassarius gayi (Kiener, 1835) & 4,8 & Frecuente & $\mathrm{FI}, \mathrm{Zl}$ \\
\hline Phidiana lottini (Lesson, 1831) & 4 & Escasa & $\mathrm{Zl}$ \\
\hline Prisogaster niger (Wood, 1828) & $2,3,4,6,8,9$ & Común & $\nabla M$ \\
\hline Purpura pansa Gould, 1853 & 14 & Rara & $\triangle M$ \\
\hline Scurria bohmita (Ramirez, 1974)** & $2,3,4,5,8,9$ & Frecuente & $\mathbb{Z M M}$ \\
\hline Scurria mesoleuca (Menke, 1851) & 16 & Rara & $\mathbb{Z M}$ \\
\hline Scurria parasitica (Orbigny, 1841) & $2,7,8,9$ & Frecuente & $\mathrm{ZMI}, \mathrm{FI}$ \\
\hline Scurria plana (Philippi, 1846) ** & 4 & Escasa & $\mathbb{Z M}$ \\
\hline Scurria variabilis (Sowerby, 1839) & 8 & Común & $\mathrm{ZMI}, \mathrm{FI}$ \\
\hline Scurria viridula (Lamarck, 1819) & $1,2,3,4,6,8,9,10$ & Frecuente & ZMS \\
\hline Scurria zebrina (Lesson, 1830) & $4,8,9$ & Escasa & $\mathrm{ZMM}, \mathrm{ZMI}$ \\
\hline Siphonaria (P.) lessoni Blainville, 1824 & $2,3,4,5,6,9$ & Frecuente & ZMS,ZMM \\
\hline Stramonita chocolata (Duclos, 1832) & $4,5,8,9,10$ & Común & $\mathrm{Zl}$ \\
\hline Stramonita haemastoma (Linnaeus, 1767) & $1,2,3,4,6,8,9,10$ & Común & $\mathrm{ZM}, \mathrm{FI}$ \\
\hline Tegula (Ch.) atra (Lesson, 1830) & $1,2,3,4,6,8,9,10$ & Común & $\mathrm{FI}$ \\
\hline Tegula (Ch.) luctuosa Orbigny, 1841 & 8 & Frecuente & Z! \\
\hline Tegula (Ch.) tridentata (Potiez \& Michaud, 1838) & $4,8,9$ & Escasa & $\mathrm{Zl}$ \\
\hline Thais (M.) triangularis (Blainville, 1832)* & 4 & Rara & $Z M$ \\
\hline Thais (Th.) callaoensis (Gray, 1828) & 9 & Escasa & $\triangle M$ \\
\hline Thais styllmanberryi Peña, 1973* & 2,3 & Escasa & $\mathrm{ZMI}, \mathrm{Fl}$ \\
\hline Trimusculus peruvianus (Sowerby, 1835) & 3 & Escasa & ZMS \\
\hline Xanthochorus buxea (Broderip, 1833) & $4,5,8,9$ & Común & $\mathrm{ZM}, \mathrm{FI}$ \\
\hline \multicolumn{4}{|l|}{ MOLLUSCA: Cephalopoda } \\
\hline Octopus mimus Gould, 1852 & $1,4,6,7,8,12$ & Común & $\mathrm{Zl}$ \\
\hline
\end{tabular}




\begin{tabular}{|c|c|c|c|}
\hline PHYLUM: Clase & Localidad” & Dominancia & Zonación \\
\hline \multicolumn{4}{|l|}{ MOLLUSCA: Bivalvia } \\
\hline Aulacomya ater (Molina, 1782) & $4,5,8$ & Escasa & $\mathrm{ZI}$ \\
\hline Barbatia (A.) pusilla (Sowerby, 1833) & 7,8 & Escasa & $\mathrm{ZMI}$ \\
\hline Brachidontes granulatus (Hanley, 1843) & 4,8 & Rara & ZMM \\
\hline Carditella (C.) tegulata (Reeve, 1843) & 8,9 & Común & $\triangle M$ \\
\hline Choromytilus chorus (Molina, 1782) & 4 & Rara & Zl \\
\hline Hiatella (H.) solida (Sowerby, 1834) & 4 & Escasa & $\mathrm{FI}$ \\
\hline Lasaea petitiana (Récluz, 1843) & $4,6,8$ & Común & $\triangle \mathrm{MM}$ \\
\hline Lithophaga (L.) peruviana (Orbigny, 1846) & 4 & Frecuente & $\mathrm{Zl}$ \\
\hline Perumytilus purpuratus (Lamarck, 1819) & $1,2,3,4,6,8,9,10$ & Dominante & ZMM \\
\hline Promantellum orbignyi Lamy, 1930 & 4 & Rara & Zl \\
\hline Protothaca $(P$.$) thaca (Molina, 1782)$ & $4,6,8$ & Frecuente & $\mathrm{Zl}$ \\
\hline Pteria sterna (Gould, 1851)* & 4 & Rara & $\mathrm{Zl}$ \\
\hline Semimytilus algosus (Gould, 1850) & $1,2,3,4,6,8,9,10$ & Dominante & $\mathrm{ZMI}$ \\
\hline \multicolumn{4}{|l|}{ ANNELIDA: Polychaeta } \\
\hline Boccardia polybranchia (Haswell, 1885) & $1,2,3,4,6,8,9,10$ & Frecuente & $\mathrm{FI}$ \\
\hline Dorvillea annulata (Moore, 1906) & $1,3,4$ & Frecuente & $2 M$ \\
\hline Halosydna johnsoni (Darboux, 1899) & $1,2,3,4,6,8,9,10$ & Común & ZMM,ZMI \\
\hline Halosydna parva Kimberg, 1856 & $1,2,3,4,6,8,9,10$ & Común & $\mathrm{ZMM}, \mathrm{ZMI}$ \\
\hline Hydroides norvegica Gunnerus, 1768 & 4 & Rara & $\mathrm{Fl}, \mathrm{Zl}$ \\
\hline Hydroides parvus (Treadwell, 1901) & 4 & Rara & $\mathrm{Fl}, \mathrm{Zl}$ \\
\hline \multicolumn{4}{|l|}{ Lepidonotus crosslandi peruana } \\
\hline Hartmann-Schröder, 1962 & 4 & Escasa & $\mathrm{FI}$ \\
\hline Lumbrineris annulata Hartmann-Schröder, 1960 & $1,2,3,4,6,8,9,10$ & Frecuente & $Z M$ \\
\hline Lumbrineris tetraura (Schmarda, 1861) & $1,2,3,4,6,8,9,10$ & Común & ZMM,ZMI \\
\hline Marphysa aenea (Blanchard, 1849) & $1,2,3$ & Frecuente & ZMM \\
\hline \multicolumn{3}{|l|}{ Hartmann-Schröder, 1962} & $\mathrm{Fl}$ \\
\hline Neanthes succinea (Frey \& Leuckart, 1847) & 4 & Rara & $\mathrm{Fl}$ \\
\hline Nereis callaona (Grube, 1857) & $1,2,3,4,6,8,9,10$ & Común & $\mathrm{ZMM}, \mathrm{ZMI}$ \\
\hline $\begin{array}{l}\text { Odontosyllis liniata Hartmann-Schröder, } 1962 \\
\text { Ophiodromus furcatus }\end{array}$ & 4,8 & Escasa & ZMM \\
\hline (Hartmann-Schröder, 1962) & $1,2,3,4,6,8,9,10$ & Frecuente & $\mathrm{ZM}$ \\
\hline Phragmatopoma moerchi Kinberg, 1867 & $1,2,3,4,6,9$ & Frecuente & $\mathrm{ZM}, \mathrm{FI}$ \\
\hline Platynereis bicanaliculata (Baird, 1863) & 4,8 & Frecuente & $\mathrm{ZM}, \mathrm{Fl}$ \\
\hline $\begin{array}{l}\text { Protoariciella uncinata Hartmann-Schröder, } 1962 \\
\text { Protomystides confusa }\end{array}$ & $21,2,3,4,6,8,9$ & Escasa & ZMM,ZMI \\
\hline Hartmann-Schröder, 1962 & 4 & Escasa & $\mathrm{ZMM}$ \\
\hline \multicolumn{4}{|l|}{ Protomystides lanceolata } \\
\hline Hartmann-Schröder, 1962 & 4 & Rara & ZMM \\
\hline Pseudonereis gallapagensis Kinberg, 1866 & $1,2,3,4,6,8,9,10$ & Común & $\mathrm{ZM}$ \\
\hline Pseudonereis variegata Grube, 1856 & $4,9,11$ & Común & ZMM,ZMI \\
\hline Sabellaria bella Grube, 1870 & 4 & Rara & $\mathrm{FI}$ \\
\hline \multicolumn{4}{|l|}{ Scoloplos armiger trioculata } \\
\hline Hartmann-Schröder, 1962 & $1,2,3,4,6,8,9$ & Común & ZMM,ZMI \\
\hline Steggoa negra Hartmann-Schröder, 1962 & $1,2,3,4,6,8,9,10$ & Común & $\mathrm{ZMM}, \mathrm{ZMl}$ \\
\hline Steggoa peruana Hartmann-Schröder, 1960 & $1,2,4,8,15$ & Frecuente & $Z M$ \\
\hline Syllis gracilis Grube, 1840 & $3,4,8$ & Frecuente & $\mathrm{ZMM}, \mathrm{ZMI}$ \\
\hline Tharyx aequiseta Hartmann-Schröder, 1962 & $3,4,5,6,8,15$ & Frecuente & $\mathrm{FI}$ \\
\hline
\end{tabular}


Tabla 1 continuación

\begin{tabular}{|c|c|c|c|}
\hline PHYLUM: Clase & Localidad & Dominancia & Zonación \\
\hline Typosyllis magdalena (Wesemberg-Lund, 1961) & $1,2,3,4,6,8,9,10$ & Común & $\mathrm{ZMM}, \mathrm{ZMI}$ \\
\hline Typosyllis prolixa Ehlers, 1901 & $1,2,3,4,6,8,9$ & Común & $\mathrm{ZMM}, \mathrm{ZMI}$ \\
\hline \multicolumn{4}{|l|}{ ARTHROPODA: Maxillopoda } \\
\hline Austromegabalanus psittacus (Molina, 1782) & $1,2,3,4,6,8$ & Común & $\mathrm{FI}, \mathrm{ZI}$ \\
\hline Balanus laevis Bruguiere, 1789 & $1,2,3,4,6,8,9,10$ & Común & $\mathrm{ZI}$ \\
\hline Jehlius cirratus (Darwin, 1854) & $1,2,3,4,6,8,9,10$ & Dominante & ZMS \\
\hline Notobalanus flosculus Darwin, 1854 & $2,3,6,8,9$ & Frecuente & $\mathrm{FI}$ \\
\hline Notochthamalus scabrosus (Darwin, 1854) & $1,2,3,4,6,8,9,10$ & Común & $\mathrm{ZMM}, \mathrm{ZM}$ \\
\hline Pollicipes elegans Lesson, 1831" & 4 & Rara & $\mathrm{Fl}$ \\
\hline Verruca laevigata (Sowerby, 1827) & $4,8,9$ & Frecuente & Zl \\
\hline \multicolumn{4}{|l|}{ ARTHROPODA: Malacostraca } \\
\hline \multicolumn{4}{|l|}{ Acanthocyclus gayi } \\
\hline (H. Milne Edwards \& Lucas, 1844) & $1,2,3,4,6,8,9,10$ & Común & $\mathrm{ZMM}, Z \mathrm{Ml}$ \\
\hline Acanthocyclus hassleri Rathbum, 1898 & 4 & Frecuente & $\triangle \mathrm{M}$ \\
\hline Acanthonyx petiverii H. Milne Edwards, 1834 & $1,4,6$ & Frecuente & $\mathrm{ZM}, \mathrm{FI}$ \\
\hline Allopetrolisthes angulosus (Guérin, 1835) & $4,6,8$ & Común & $\mathrm{ZMI}, \mathrm{FI}$ \\
\hline Allopetrolisthes punctatus (Guérin, 1835) & 4,6 & Escasa & $\mathrm{Fl}$ \\
\hline \multicolumn{4}{|l|}{ Allopetrolisthes spinifrons } \\
\hline (H. Milne Edwards, 1837) & 4,8 & Frecuente & ZI \\
\hline Alpheus chilensis Coutiere, 1902 & 4,8 & Rara & $\mathbb{Z M}$ \\
\hline Alpheus inca Wicksten \& Mendez, 1981 & 4 & Rara & $\mathbb{Z M}$ \\
\hline Betaeus emarginatus ( $H$. Milne Edwards, 1837) & 4,8 & Rara & $\mathbb{Z M}$ \\
\hline Betaeus truncatus Dana, 1852 & 4,8 & Rara & $\mathbb{Z M}$ \\
\hline Cancer porteri Rathbun, 1930 & 4 & Escasa & $\mathrm{Fl}, \mathrm{ZI}$ \\
\hline Cancer setosus Molina, 1782 & 1,4 & Frecuente & $\mathrm{Fl}, \mathrm{Zl}$ \\
\hline \multicolumn{4}{|l|}{ Caprella cf. pilipalma } \\
\hline Dougherty \& Steinberg. 1953 & $7,9,15$ & Frecuente & ZMM \\
\hline Cyclograpsus cinereus Dana, 1851 & 4,8 & Frecuente & ZMS,ZMM \\
\hline \multicolumn{4}{|l|}{ Cycloxanthops sexdecimdentatus } \\
\hline (H. Milne Edwards \& Lucas, 1843) & 4 & Escasa & $\mathrm{Fl}, \mathrm{Zl}$ \\
\hline Dynamenella bakeri (Menzies, 1962) & $1,2,3,4,6,8,9$ & Común & ZMM,ZM! \\
\hline Elasmopus cf. rapax Costa, 1853 & $1,2,3,4,6,8,9,10$ & Común & $Z M M, Z M I$ \\
\hline Eurypanopaeus transversus (Stimpson, 1860) & 4 & Escasa & $\mathrm{ZI}$ \\
\hline \multicolumn{4}{|l|}{ Gaudichaudia gaudichaudii } \\
\hline (H. Milne Edwards, 1834) & 4,8 & Escasa & $\mathrm{Fl}$ \\
\hline Grapsus grapsus (Linnaeus, 1758) & $1,2,3,4,6,8,9,10$ & Común & FS,ZMS \\
\hline Liopetrolisthes mitra (Dana, 1852) & $4,6,8$ & Frecuente & $\mathrm{F}\}, \mathrm{Zl}$ \\
\hline Pachycheles crinimanus Haig, 1960 & $1,3,4,6,8$ & Común & $\mathrm{FI}, \mathrm{ZI}$ \\
\hline Pachycheles grossimanus (Guérin, 1835) & 4 & Frecuente & $\mathrm{FI}, \mathrm{Zl}$ \\
\hline Pachygrapsus transversus (Gibbes, 1850) & $1,2,3,4,6,9$ & Frecuente & ZMS,ZMM \\
\hline Petrolisthes desmarestii (Guérin, 1835) & 4 & Rara & ZMI \\
\hline Petrolisthes granulosus (Guérin, 1835) & 4,8 & Común & ZMS \\
\hline Petrolisthes laevigatus (Guérin, 1835) & 4 & Frecuente & ZMS \\
\hline Petrolisthes tuberculatus (Guérin, 1835) & 4 & Escasa & $\mathrm{ZMl}$ \\
\hline Petrolisthes violaceus (Guérin, 1831) & $4,6,8,9$ & Común & $\mathrm{ZMI}, \mathrm{FI}$ \\
\hline Pilumnoides periatus (Poeppig, 1836) & $3,4,8$ & Frecuente & $\mathrm{ZMI}, \mathrm{Fl}$ \\
\hline
\end{tabular}


Tabla 1 continuación

\begin{tabular}{|c|c|c|c|}
\hline PHYLUM: Clase & Localidad ${ }^{\mathrm{B}}$ & Dominancia & Zonación \\
\hline Pinnaxodes chilensis ( $\mathrm{H}$. Milne Edwards, 1837) & $6,7,8,9$ & Común & $\mathrm{Zl}$ \\
\hline Pinnaxodes silvestrii Nobili, 1901 & 9 & Escasa & $\mathrm{FI}$ \\
\hline Pinnotheres ostreum Say, 1817 & 4 & Frecuente & $\mathrm{Zl}$ \\
\hline \multicolumn{4}{|l|}{ Plalyxanthus orbignyi } \\
\hline (H. Milne Edwards \& Lucas, 1843) & $1,4,8$ & Rara & $\mathrm{Fl}, \mathrm{ZI}$ \\
\hline Rhynchocinetes typus H. Milne Edwards, 1837 & 4 & Rara & $\mathbb{Z M}$ \\
\hline Synalpheus spinifrons ( $\mathrm{H}$. Milne Edwards, 1837) & 4,8 & Rara & $\mathbb{Z M}$ \\
\hline Teleophrys tumidus (Cano, 1889) & 4 & Rara & $\mathrm{Zl}$ \\
\hline \multicolumn{4}{|l|}{ ECHINODERMATA: Asteroidea } \\
\hline Luidia magellanica Leiplodt, 1895 & 4,8 & Escasa & $\mathrm{FI}, \mathrm{ZI}$ \\
\hline Heliaster helianthus (Lamarck, 1816) & $1,2,3,4,6,8,9,10$ & Común & $\mathrm{ZM}, \mathrm{FI}$ \\
\hline Patiria chilensis (Lütken, 1859) & $1,2,3,4,6,8,9,10$ & Común & $\mathrm{ZMI}, \mathrm{FI}$ \\
\hline Stichaster striatus Müller \& Troschel, 1840 & $1,2,3,4,6,8,9,10$ & Común & $\mathrm{ZMI}, \mathrm{FI}$ \\
\hline \multicolumn{4}{|l|}{ ECHINODERMATA: Ophiuroidea } \\
\hline Ophiacthis kröyeri Lütken, 1856 & $1,2,3,4,6,8,9,10$ & Común & $\mathrm{ZM}, \mathrm{FI}, \mathrm{Zl}$ \\
\hline \multicolumn{4}{|l|}{ ECHINODERMATA: Echinoidea } \\
\hline Arbacia incisa (Agassiz, 1863) & $1,2,3,4,6,8,9,10$ & Común & $\mathrm{Fl}, \mathrm{Zl}$ \\
\hline Arbacia spatuligera (Valenciennes, 1846) & $4,7,8$ & Escasa & $\mathrm{FI}, \mathrm{ZI}$ \\
\hline \multicolumn{4}{|l|}{ Caenocentrotus gibbosus } \\
\hline (Agassiz \& Desor, 1846) & $4,6,7,8$ & Común & $\mathrm{Zl}$ \\
\hline Tetrapigus niger (Molina, 1782) & $2,3,4,6,8,9$ & Escasa & $\mathrm{Fl}, \mathrm{Zl}$ \\
\hline \multicolumn{4}{|l|}{ ECHINODERMATA: Holothuroidea } \\
\hline Athyonidium chilensis (Semper, 1868) & 4,9 & Escasa & $\mathrm{Fl}$ \\
\hline Pattalus mollis Selenka, 1868 & $1,2,3,4,6,8,9,10$ & Común & $\mathrm{Fl}$ \\
\hline
\end{tabular}

Tabla 2. Localidades representativas del Departamento de Lima, Perú, indicando tipo de orilla y número de especies.

\begin{tabular}{lclc}
\hline \multicolumn{1}{c}{ LOCALIDAD } & LATITUD $(S)$ & TIPO DE ORILLA & N $^{\circ}$ ESPECIES \\
\hline Chancaíllo & $11^{\circ} 29^{\prime} 27^{\prime \prime}$ & Expuesta & 60 \\
Ancón & $11^{\circ} 46^{\prime} 33^{\prime \prime}$ & Protegida & 151 \\
Pucusana & $12^{\circ} 28^{\prime} 57^{\prime \prime}$ & Semiexpuesta & 113 \\
Asia & $12^{\circ} 57^{\prime} 39^{\prime \prime}$ & Muy expuesta & 79 \\
\hline
\end{tabular}

los Phyla indicados en la Figura 2, entre las que destacan los moluscos (73 especies), poliquetos ( 31 especies) y crustáceos (30 especies). El total de las especies del intermareal rocoso citadas para Pisco, es similar al total de los tres taxa hallados por nosotros en el Departamento de Lima.
En la orilla rocosa se observa una alta diversidad, sobre todo en las comunidades de mitílidos y en la comunidad de Phragmatopoma moerchi, en las que se ha registrado hasta 87 especies, excluyendo los briozoos encostrantes y los nemátodos (Pardo, 1975; Paredes \& Tárazona, 1980). 
La diversidad de invertebrados en la orilla y los fondos rocosos también ha sido estudiada en el extremo sur de la Provincia Peruana, Chile (Zamorano y Moreno, 1975; López y Osorio, 1977; Romo y Alveal, 1977, Peña y Clarke, 1983), observándose una menor diversidad de especies a medida que se avanza hacia latitudes más altas.

Teniendo en cuenta nuestros resultados y las observaciones en diversas localidades (datos no publicados) entre Bahía de Ferrol, Chimbote $\left(09^{\circ} \mathrm{S}\right)$ y Vila Vila, Tacna $\left(18^{\circ} \mathrm{S}\right)$, podemos afirmar que la diversidad de grupos taxonómicos y la distribución vertical de las especies en la zona intermareal rocosa presenta un mismo patrón, el cual podría ser generalizado para toda la Provincia Peruana.

\section{LITERATURA CITADA}

Huamán, P. 1974. Estudio de los Polyplacophora (Phylum Mollusca) del litoral del Departamento de Lima, con especial referencia de la rádula. Tesis de Bachiller. UNMSM. Lima, Perú. 49 pp.

López, M., y C. Osorio. 1977. Diversidad biológica en una comunidad intermareal de Putemun. Chiloe. Bol. Soc. Biol. de Concepción, 51 (1): 123-127.

Pardo, J. 1975. Contribución al conocimiento de la comunidad de Phragmatopoma peruensis Hartman, 1944 (Polychaeta, Sabellariidae). Tesis de BachiIler. UNMSM. Lima, Perú. 35 pp.

Paredes, C. 1974a. Contribución al conocimiento de los invertebrados del litoral rocoso del Departamento de Lima con estudio especial de la biocenosis de mitílidos. Tesis doctoral. UNMSM. Lima, Perú. 141 pp.

Paredes, C. 1974b. El modelo de zonación en la orilla rocosa del Departamento de Lima. Rev. Per. Biol. I(2): |68-191
Paredes, C. 1980. La familia Acmaeidae (Gastropoda, Archaeogastropoda) en el Perú. Rev. Per. Biol. 2( I): $52-58$

Paredes, C. 1986. La familia Fissurellidae (Gastropoda, Archaeogastropoda) en el Perú. Revista de Ciencias UNMSM, 74(1): 75-86.

Paredes, C. y J. Tarazona. 1980. Las comunidades de mitílidos del mediolitoral rocoso del Departamento de Lima. Rev. Per. Biol. 2(1): 59-71.

Paredes, C. y J. Tarazona, E. Canahuire, L. Romero y O. Cornejo. 1988. Invertebrados macrobentónicos del área de Pisco, Perú. En: H. Salzwedel y A. Landa (eds.). Recursos y dinámica del ecosistema de afloramiento peruano. Bol. Inst. Mar PerúCallao, Vol. Extraordinario: 121-132.

Peña, R. y M. Clarke. 1983. Estudio de la Comunidad de Perumytilus purpuratus (Lamarck, 1819) en el intermareal rocoso de Antofagasta (II Región, Chile). Instituto de Investigaciones Oceanológicas. Universidad de Antofagasta. Chile. 13 p. (MS)

Romo, H. y K. Alveal. 1977. Las comunidades del litoral rocoso de Punta Ventanilla, Bahia de Quintero, Chile. Gayana Misc. 6: 1-40.

Tait, R. V. 197I. Elementos de Ecología Marina. Ed. Acribia, Zaragoza, España, 320 pp.

Tarazona, J. 1974. Poliquetos errantes de la zona litoral del Departamento de Lima. Tesis de Bachiller. UNMSM. Lima, Perí. 97 pp.

Tarazona, J. y S. Valle. 1998. La diversidad biológica en el mar Peruano. En: G. Haffter (comp.). La diversidad biológica de Iberoamérica III. Acta Zoologica Mexicana, nueva serie, Vol. especial: 103-II5

Tarazona, J., C. Paredes, L. Romero, V. Blaskovich, S. Gúzman y S. Sánchez. 1985. Características de la vida planctónica y colonización de los organismos bentónicos epilíticos durante el Fenómeno "El Niño". En: Arntz, W., A. Landa y J. Tarazona (eds.). "El Niño": Su impacto en la fauna marina. Bol. Inst. Mar. Perú-Callao, Vol. Extr.; 4I-49.

Zamorano, J. y C. Moreno. 1975. Comunidades bentónicas del sublitoral rocoso de Bahía Corral. I. Area mínima de muestreo y descripción cuantilativa de la asociación de Pyura chilensis Molina. Medio Ambiente, I(1): 58-66. 